Para enlazar con este artículo / To link to this article:

http://dx.doi.org/10.6035/MonTI.2019.ne4.6

Para citar este artículo / To cite this article:

Martínez Pleguezuelos, Antonio J. \& J. David González-Iglesias. (2019) "Identidades presas: representación, estereotipo e interseccionalidad en la traducción de la mujer latina en Orange Is The New Black." En: Pérez L. de Heredia, María \& Irene Higes Andino (eds.) 2019. Multilingüismo y representación de las identidades en textos audiovisuales / Multilingualism and representation of identities in audiovisual texts. MonTI Special Issue 4, pp. 173-198.

\title{
IDENTIDADES PRESAS: REPRESENTACIÓN, ESTEREOTIPO E INTERSECCIONALIDAD EN LA TRADUCCIÓN DE LA MUJER LATINA EN ORANGE IS THE NEW BLACK ${ }^{1}$
}

\author{
Antonio J. Martínez Pleguezuelos \\ ajesus.martinez@usal.es \\ Universidad de Alcalá \\ J. David González-Iglesias \\ david.gonzalez.iglesias@uam.es \\ Universidad Autónoma de Madrid
}

\section{Resumen}

En la siguiente investigación nos aproximamos a la traducción de productos audiovisuales como vía para construir identidades colectivas e individuales. Partimos de la premisa de que, en la época global que vivimos, los medios audiovisuales (traducidos) contribuyen a la formación de identidades interseccionales atravesadas por rasgos como la edad, el género, la raza o la sexualidad. Por ello, como mantendremos, resulta necesario entender el discurso traducido como parte constituyente del tejido social. Para dar prueba de ello estudiaremos el caso del grupo de presas latinas de la serie Orange Is the New Black, especialmente los rasgos de la sexualidad y el uso del español con el fin de comprobar qué asimetrías surgen en la recreación de esta minoría para los espectadores en la versión original y en la doblada.

1. El presente artículo se inscribe en el proyecto de investigación «Violencia simbólica y traducción: retos en la representación de identidades fragmentadas en la sociedad global» (FFI2015-66516-P; MINECO / FEDER, UE), financiado por el Ministerio de Economía y Competitividad del Gobierno de España y el Fondo Europeo de Desarrollo Regional. 


\begin{abstract}
"Prisoner Identities: Representation, Stereotype and Intersectionality in the Translation of the Latina Woman in Orange Is the New Black"

In this study we approach the translation of audiovisual products as a way to construct collective and individual identities. We start from the premise that in the global era in which we live, (translated) audiovisual media contribute to the formation of intersectional identities characterized by features such as age, gender, race or sexuality. For this reason, it becomes necessary to understand translated discourse as an integral part of the social fabric. To support this statement, we study the case of the group of Latina inmates in the series Orange Is the New Black, particularly with regard to their sexuality and use of Spanish, in order to verify what asymmetries emerge from the re-creation of this minority for viewers in the original and dubbed versions of the series.
\end{abstract}

Palabras clave: Traducción. Identidad. Interseccionalidad. Orange Is the New Black. Mujeres latinas.

Keywords: Translation. Identity. Intersectionality. Orange Is the New Black. Latina women. 


\section{Introducción}

Dentro del caudal transnacional imparable de bienes y servicios, las imágenes, fotogramas y diálogos que se emiten a millones de espectadores a través de los medios de comunicación actuales en cualquier parte del mundo han contribuido al declive de paradigmas hegemónicos y monolingües en las series de ficción televisadas en favor de un crisol catódico de razas, géneros, acentos, sexualidades, lenguas y demás rasgos que contribuyen a la definición de las distintas identidades proyectadas.

La representación de los personajes se convierte así en un reflejo fiel en la pantalla de televisión de la realidad plural, heteroglósica, híbrida, multicultural y multilingüe que hoy en día es frecuente en las sociedades que vivimos. No por casualidad, Castells afirma en su estudio sobre la comunicación y el poder en los mass media que lo que no existe en los medios no existe para el público general, por lo que el contenido transmitido será, irremediablemente, un mensaje mediático (2008: 17). Con ello, continúa, "los medios de comunicación de masas no son los depositarios del poder, pero en conjunto constituyen el espacio en el que se decide el poder" (2008: 17), argumento que concuerda con el de distintas voces desde los Estudios Culturales (Hall 1973; Chambers 2009) cuando afirman que la televisión, como cualquier otro medio de comunicación cultural, participa en la construcción de nuestra realidad.

Con estas premisas en mente y amparados bajo las últimas propuestas de los estudios de Traducción Audiovisual que incluyen las aportaciones del Giro Cultural (cf. Díaz-Cintas 2012a, 2012b; de Marco 2012; Pérez L. de Heredia 2015, 2016a, 2016b; Díaz-Cintas, Parini y Ranzato 2016), emprendemos la siguiente investigación siendo conscientes de que, en esta era de la hiperconexión global, el trasvase informativo que se lleva a cabo en los productos culturales traducidos es mediático y, al tiempo, estos "son y están significativamente mediados" (Martín Ruano 2016: 2).

Para dar cuenta de los nuevos modelos de ficción televisada (GonzálezIglesias y Toda 2013) y del potencial de la traducción a la hora de (re)construir identidades híbridas, nos aproximamos en las siguientes páginas a una de las series televisivas que, en la actualidad, muestra de manera más clara 
la diversidad identitaria en todas sus vertientes: Orange Is the New Black (OITNB) (Netflix 2013). Bajo la dirección de Jenji Kohan y producida por Tilted Productions en colaboración con Lionsgate Television, esta serie estadounidense se estrenó en julio de 2013 a través de la plataforma de streaming Netflix y cuenta, hasta este momento, con cuatro temporadas y la confirmación de la quinta para 2017. En ella, mujeres de orígenes, razas, etnias, clases sociales, sexualidades, culturas e idiomas distintos conviven en el microcosmos televisivo de la ficticia prisión estadounidense de Litchfield, donde se desarrolla gran parte de los diferentes arcos argumentales. Si bien las tramas que presentan a los distintos grupos de presas han sido elogiadas por crítica y público por superar muchos estereotipos inscritos durante años en cuerpos que no encajaban en el paradigma blanco, heterosexual y cisgénero de clase media-alta que solía protagonizar los productos de ficción hasta hace relativamente poco tiempo (Rosenberg 2015), reparamos en que, en cierta medida, los creadores de la serie continúan mostrando a la comunidad latina desde un encuadre que no captura en pantalla perfiles sexuales divergentes.

Este análisis se ha llevado a cabo a través de una metodología descriptiva y empírica. Mediante el visionado de la serie y el estudio comparado de las soluciones de doblaje en la versión emitida para el público español y el original hemos seleccionado aquellas escenas que, por su carga lingüística o de representación de diferentes identidades, ilustran con claridad los distintos aspectos relacionados con el estereotipo y la interseccionalidad que planteamos en nuestro análisis. Debido a las obvias restricciones de espacio, no es esta una lista exhaustiva de casos, sino una muestra que nos guía a través del estudio de la representación en un entorno globalizado. Por tratarse de textos audiovisuales, somos conscientes de las limitaciones técnicas que subyacen a este tipo de traducción subordinada. Sin embargo, no es nuestra intención detenernos en la observación de las características microtextuales de las escenas que presentamos, sino comprobar qué imagen se presenta al espectador español de la comunidad latina en OITNB y qué repercusiones tendrá. Para ello partiremos desde los postulados de los Estudios Culturales (Hall 1996, 2000), que entienden la identidad de cada sujeto como un producto creado de manera discursiva. En nuestro caso, nos interesará retomar esta perspectiva porque, en consonancia con distintos teóricos en el campo de los Estudios de Traducción (House, Martín Ruano y Baumgarten 2005; Cronin 2006; Vidal Claramonte 2007), consideramos la identidad como una construcción expuesta a la reescritura, y así lo comprobaremos a través de la comparación de una serie de ejemplos de las versiones original y traducida de OITNB. 
De manera específica, en la siguiente investigación nos plantearemos cómo se ha representado en la versión en español la intersección entre raza y sexualidad en el grupo de latinas de OITNB a través de ejemplos concretos extraídos de distintos capítulos. Para ello, y considerando los objetivos establecidos, estudiaremos el uso del español como marca de latinidad y las consecuencias que derivan de su alternancia con el inglés para las identidades representadas. Asimismo, observaremos si este rasgo de latinidad ha superado paradigmas de productos de ficción de décadas anteriores en los que las sexualidades minoritarias no tenían cabida. Por tanto, será necesario acudir también a los Estudios de Género y Sexualidad para llevar a cabo una investigación interdisciplinar que nos permita entender la complejidad de la labor del traductor audiovisual al (re)escribir identidades interseccionales (Brufau 2009, 2010; von Flotow 2009). Con ello, concluiremos que a pesar de los indudables avances que se han producido en la representación identitaria mediante el discurso audiovisual traducido, especialmente en la serie que nos ocupa, existen todavía dificultades a la hora de representar y reescribir personajes interseccionales sin caer en estereotipos o sin crear una imagen modificada para el público meta.

\section{Las identidades mediáticas (y mediadas) en los productos de ficción globalizados}

Hall (1973) describió cómo se codifica y descodifica la información transmitida a través de los medios de comunicación de masas. En su influyente investigación, este autor explica cada uno de los pasos del proceso seguidos para comunicar mediante las imágenes, los diálogos y las diferentes escenas que se suceden en cualquier producto de ficción. Como parte de su argumentación, Hall incide en el contexto social que envuelve al espectador y en el poder de la audiencia para descodificar mensajes en función de su entorno cultural y social, entendiendo así que el papel activo del público se convierte en una puerta abierta para el cambio social.

Ante este planteamiento, el traductor, como primer y experimentado espectador, contribuye a la construcción de la identidad cultural de diversas comunidades; por ello, no es casualidad que Gentzler (2012: s/p) señale que "translation [...] becomes the fundamental process that allows the very existence of any signifying activity". Las vías audiovisuales son un ejemplo paradigmático de ello, y queda demostrado por el creciente número de investigaciones y publicaciones que se aproximan a textos audiovisuales desde enfoques que incluyen los postulados del giro cultural. No obstante, como apunta Díaz-Cintas (2012a: 275), aún queda mucho camino por recorrer en el análisis de las consecuencias derivadas del poder, la ideología, la censura o la 
manipulación en la reescritura de productos audiovisuales, lo que supone un acicate para, en sus palabras, "find synergies with other disciplines and apply the same rigorous interrogation that other areas of translation have recently undergone".

La traducción supera en el ámbito audiovisual los límites de lo lingüístico para conjugar de manera discursiva orígenes múltiples en espacios de convivencia fragmentados donde las diferencias identitarias entre los distintos sujetos condicionan las vías de representación de la multiculturalidad en la ficción. Así, desde la visión cosmopolita que se impone con fuerza en nuestras sociedades, coincidimos con Cronin (2006: 10) cuando defiende "the possibility of thinking about translation as a way not only of thinking but of being and acting in the world". De hecho, en consonancia con diversos autores que abundan en la condición en traducción del sujeto (cf. Vidal Claramonte 2007: 41), Cronin (2006: 45) mantiene que:

translation takes place in the physical sense of movement or displacement and in the symbolic sense of the shift from one way of speaking, writing about and interpreting the world to another.

Los personajes que participan en OITNB son una clara muestra de este panorama multicultural que describimos. La narración de la serie, que se presenta en un primer momento desde la perspectiva de Piper Chapman, una mujer joven, blanca y de clase media-alta que entra en prisión por tráfico de drogas, amplía pronto el foco para mostrar el crisol de culturas, razas, credos y, en suma, identidades, que conviven en la penitenciaría de Lichtfield. De este modo, deberíamos considerar, en sintonía con las palabras de Shohat y Stam (2003: 4), que "it is perhaps time to think in terms of comparative and transnational multiculturalism, of relational studies that do not always pass through the putative center" con el propósito de dar voz y visibilidad a ciertas identidades interseccionales que han permanecido ocultas en la ficción hasta hace relativamente pocos años.

De forma paralela, Hall (2000: 596) alude al cambio estructural que está modificando las sociedades contemporáneas desde los últimos años del siglo XX a través de una "descentralización" de la identidad. Esta "dislocación" a la que se refiere este autor describe de manera crítica e integradora los nuevos modelos identitarios de sociedades plurales que, a pesar de estar aún infrarrepresentados en la ficción actual, van consiguiendo minutos de pantalla en las nuevas producciones (traducidas). En este juego de representaciones identitarias son distintas las voces que reclaman una mayor atención al poder de la televisión en tanto que potente canal para moldear identidades colectivas y 
multilingües por su enorme capacidad para visibilizar y normalizar paradigmas minoritarios. Así lo explica Hall (1995: 90) cuando defiende que:

In modern societies, the different media are especially important sites for the production, reproduction and transformation of ideologies. [...] Institutions like the media are peculiarly central to the matter since they are, by definition, part of the dominant means of ideological production.

Para el desarrollo de esta línea teórica y desde el enfoque que asumimos en esta investigación, nos interesa especialmente el carácter performativo del lenguaje, como lo concibe Butler (1990), a la hora de construir las identidades individuales y colectivas de los productos de ficción. Esta autora acude a la fuerza de la representación lingüística que moldea la realidad y plantea que la representación del sujeto sólo puede darse en lo que ya se conoce como tal (1990). Las acciones discursivas legitimadoras y excluyentes a las que se refiere Butler dan forma al sujeto, penetran en la sociedad y la cultura y quedan, de esta manera, naturalizadas. Así, el poder produce lo que en principio parece sólo representar (Butler 1990) y ahí reside, de acuerdo con esta argumentación, el peligro de considerar que el sujeto y, con ello, la identidad, anteceden al lenguaje. De esta forma, la identidad que concibe Butler en clave postestructuralista aparece como el efecto del lenguaje y no como una idea previa, exterior e independiente de él. Con ello, insiste la autora, tendremos que prestar atención a la construcción discursiva inestable que abre el camino a significaciones condicionadas por la interseccionalidad de distintos contextos históricos y culturales que, a su vez, darán lugar a nuevas resignificaciones.

En el caso de OITNB, el carácter performativo que retomamos es fundamentalmente apreciable por la organización social de las presas. La cárcel de Litchfield se encuentra dividida en grupos constituidos en torno a un rasgo identitario común para cada uno de ellos: las internas blancas, negras y latinas conviven en un orden jerárquico perfectamente delimitado que da lugar a muchas de las escenas que se suceden en la serie. Como señalan Enck y Morrissey (2015: 309), muchas de las situaciones cómicas o de tensión que se producen entre las reclusas se dan cuando alguna de ellas traspasa una de esas fronteras y pone en juego el precario equilibrio social que rige su día a día. La división de las comunidades de presas en OITNB se refleja desde los primeros capítulos y tiene un corte, como apuntábamos, principalmente racial. De este modo, cada grupo se constituye en torno a los distintos dormitorios donde residen dentro de prisión: las presas blancas se encuentran en "The Suburbs", las negras en "The Ghetto" y las latinas en el "Spanish Harlem". En los siguientes ejemplos comprobamos cómo las propias reclusas hacen referencia a la asignación de las celdas y a la compartimentación en grupos aislados cuando, 
en los primeros episodios, la protagonista, Chapman, se encuentra a la espera de asignación de su celda junto con dos veteranas de la prisión, Nicky y Anita:

Ejemplo 1. OITNB 01x03 (05:172)

CHAPMAN: Why didn't I get assigned?

NICKY: They're probably sending her to Spanish Harlem. They're gonna put you in

The Suburbs with the other white people.

CHAPMAN: So how do they choose your roommate?

NICKY: Why? You afraid you're gonna end up with your rocka-lezzie girlfriend?

CHAPMAN: ¿A mí por qué no me dan?

NICKY: A esta la mandarán al Spanish Harlem y a ti a un barrio residencial con el resto de las blancas.

CHAPMAN: ¿Y cómo eligen a tu compañera?

NICKY ¿Por qué? ¿Te da miedo acabar con tu amiga la bollera?

Ejemplo 2. OITNB 01x03 (51:23)

GUARDA: Chapman! Get your things together. You're moving down to B dorm. Cube 18 !

ANITA: That's the ghetto.

CHAPMAN: What?

ANITA: They got you in the ghetto. Good luck, Chapman. You're gonna need it.

GUARDA: Chapman, recoge tus cosas. Te trasladas al dormitorio B. Cuarto 18.

ANITA: Es el gueto.

CHAPMAN: ¿Qué?

ANITA: Te han metido en el gueto. Buena suerte, Chapman, la necesitarás.

A pesar de que todas se organizan en grupos más o menos compartimentados, la novedad de este drama carcelario reside en el carácter interseccional que se observa en muchas de las protagonistas. Además de la perspectiva de género, cada una de ellas se encuentra atravesada por otras circunstancias, como la raza, la identidad sexual, la adicción a distintas sustancias, el uso de distintas lenguas para comunicarse o la edad. De hecho, una de las características que marca la formación de uno de los grupos en la serie es este último rasgo, lo que hace que, junto a las latinas, las negras y las blancas, encontremos también a las "Golden Girls" o "chicas de oro", a las que se refiere una de las reclusas de forma explícita durante una conversación en la que Chapman, recién llegada

2. Los ejemplos que se presentan en este artículo muestran, salvo indicación expresa, la transcripción del diálogo en versión original y, a continuación, la transcripción del doblaje al castellano. El código de identificación de temporadas y capítulos en el pie de cada uno de los ejemplos sigue el formato YYxZZ, donde "YY" es el número de temporada y "ZZ" el número de capítulo. 
a la cárcel, trata de entender cuáles son las dinámicas de grupo que existen en su nuevo entorno:

Ejemplo 3. OITNB 01x06 (18:02)

CHAPMAN: So, who are you running against besides Taystee?

MORELLO: She don't count. Black ladies just run against the other black ladies. My competition is Pennsatucky. But it don't matter, because Red's gonna make all the white girls vote for me.

CHAPMAN: So, you only run against white people.

NICKY: You can only vote within your race or your group. Look, just pretend it's the 1950s. It makes it easier to understand.

MORELLO: See, everyone elects a representative from their own tribe. White, black, Hispanic, golden girls, others. And those five gals, they meet with Healy, they tell him what we want, then he speaks to the higher-ups. It's like student council.

CHAPMAN: ¿Y a quien te enfrentas aparte de a Taystee?

MORELLO: Ella no cuenta. Las negras solo compiten con otras negras. Yo tengo que ganar a Pennsatucky, pero no me preocupa porque Red obligará a todas las blancas a votarme.

CHAPMAN: ¿Entonces solo te enfrentas a otras blancas?

NICKY: Solo puedes votar a alguien de tu raza o tu grupo. Piensa que estamos en 1950 y lo entenderás.

MORELLO: Verás, se elige a un representante de cada tribu: blancas, negras, hispanas, chicas de oro y otras. Luego las cinco chicas se reúnen con Haley y le dicen lo que queremos y así él habla con los jefazos. Como los delegados de clase.

En este producto penitenciario rodado en clave feminista, los cuerpos se encuentran sujetos a diversos condicionantes. Lejos quedan las ficciones en las que la male gaze que definía Mulvey (1975) dibujaba perfiles femeninos condicionados por un punto de vista patriarcal. En consonancia con otras grandes producciones, OITNB muestra a la "nueva mujer" que Pérez L. de Heredia (2016b: 170) describe, alejando a los personajes femeninos del estereotipo que los sitúa en un segundo plano, indefensos y con escasa relevancia. Este nuevo papel protagonista viene marcado por un fuerte carácter interseccional (Brufau 2009: 19) en el que cada uno de los grupos dentro de la prisión presenta reclusas "atravesadas", en terminología de Vidal Claramonte (2015), por características que las diferencian entre sí. Así, la segmentación racial que se produce en la cárcel no se asimila necesariamente con una división religiosa uniforme. Por ejemplo, la llegada de una nueva remesa de reclusas en la cuarta temporada de la serie aumenta las fricciones entre las protagonistas, como se ilustra en el caso de Cindy, del subgrupo de presas negras, que se convierte del catolicismo al judaísmo dentro de la prisión, pasa a llamarse Tova y recibe una nueva compañera de celda, Alison, también negra, que lleva puesto el velo islámico. Desde un punto de vista meramente lingüístico, su primer contacto, 
muy breve, resulta bastante esclarecedor. Tiene lugar cuando Alison llega a la celda por primera vez:

Ejemplo 4. OITNB 04x01 (38:07)

\begin{tabular}{|l}
\hline CINDY: Shalom. \\
ALISON: Assalam-alaikum. \\
\hline CINDY: Shalom. \\
ALISON: Assalam-alaikum.
\end{tabular}

La perspectiva interseccional adquiere, como observamos, especial relevancia en los productos multilingües por el potencial que presenta en la construcción de personajes complejos. En el caso concreto al que dedicamos las siguientes páginas reparamos en que, hasta hace relativamente poco tiempo, las series de ficción empleaban un rango limitado de arquetipos para recrear la identidad latina, arquetipos construidos sobre estereotipos preconcebidos y muy negativos que infrarrepresentaban a dicha comunidad, al estilo de cómo Morello, una de las presas de la serie, se refiere a los latinos: "They live like 20 people in one apartment. They have more kids that even the Irish, the men like their women with big titties and big asses, they're dirty, they're greasy, their food smells nasty, and they're taking a lot of jobs" (episodio 1x06). Como argumenta Vidal Claramonte (2007: 29):

[e]l estereotipo reduce y exagera, pero sobre todo inmoviliza, fija unos rasgos, los naturaliza; convierte lo diferente en anormal, exótico, temible y, muchas veces, inaceptable, por lo que establece límites y procedimientos de exclusión.

Esto, proyectado a través de los medios audiovisuales y de su traducción, multiplica exponencialmente su alcance alrededor del mundo. En el caso específico de OITNB, los estereotipos se emplean en muchos casos como punto de partida para presentar las distintas identidades colectivas y para, en última instancia, superar ideas e imágenes preconcebidas sobre las minorías raciales y sexuales representadas, como pasamos a analizar a continuación.

\section{Representación y traducción de la identidad latina en OITNB y la (no) interseccionalidad}

Ante las dificultades que plantea la traducción de productos de ficción globalizados, y en función de las premisas que hemos delineado, cabe plantearse ahora, de la mano de Pérez L. de Heredia (2016a: 194), si

será la nueva interseccionalidad representada en la ficción televisiva el desafío definitivo e irreversible que acabe con la cultura mainstream masculina, blanca 
y protestante que ha colonizado pantallas e idearios del mundo occidental [y si] será capaz de transformarla en un retrato polícromo, inclusivo en términos de género y raza, acorde con la nueva realidad mundial.

El reto no es cuestión baladí si recordamos el poder de la traducción para (re) crear el tejido cultural y social de una comunidad (Gentzler 2012), especialmente ante el alegato de distintas voces desde los Estudios Culturales cuando afirman que el aspecto de la producción cultural y de la creación de discursos políticos parece decidirse hoy más que nunca en zonas híbridas de fricción, de superposición y de contacto.

En OITNB, crítica y público parecen estar de acuerdo en que la representación del grupo de presas llevada a cabo en la serie escapa de imágenes esencializadas y supera los sistemas clasificatorios estancos de género, sexualidad y raza en favor de concepciones mutables y fluidas que cambian en función de las fuerzas de poder sociales y culturales (Rosenberg 2015). Su creadora, Jenji Kohan, no ignora el potencial que guardan las protagonistas, ricas en matices, y profundiza en las interrelaciones que se suceden entre las diferentes reclusas al tiempo que explora las superposiciones de las distintas facetas identitarias de cada personaje. No todas las reclusas son malas, no todas son blancas, no todas se reconocen dentro del binomio heterosexual/homosexual y, por supuesto, no todas son monolingües. Por ello el tipo de representación que se hace de cada personaje cobra importancia y se hace necesario un análisis desde un marco de trabajo que analice las narrativas construidas alrededor de cada grupo de reclusas.

Para el análisis específico de los procesos de traducción que afectan al grupo de presas latinas hemos de tener presentes los estereotipos a los que los guionistas recurrían en televisión hasta hace muy pocos años para representar a los personajes de origen hispano. Tal y como describe Valdivia (2014: 20), los tropos más frecuentes eran, por una parte, el de la madre de familia abnegada dedicada por completo a sus hijos y a su matrimonio; el de asistenta doméstica al servicio de una familia adinerada; y, en el otro extremo, el de la mujer sexualizada y provocativa, connotado, en la mayor parte de los casos, de manera muy negativa. A continuación, será necesario contar con estas representaciones en los ejemplos extraídos de OITNB para comprobar si se han superado viejos arquetipos para su representación y su traducción o si, por el contrario, siguen predominando en la nueva ficción.

Hemos de tener en cuenta además que, en este reconocimiento interseccional de las identidades televisadas, el multilingüismo se convierte en otra característica más del personaje especialmente relevante, que lo define de un modo muy concreto y que, por descontado, no pasará desapercibido para el 
espectador. El encuentro y reconocimiento de una raza, una lengua, una sexualidad y un género específicos reflejan la complejidad de los nuevos paradigmas en la ficción actual. En el primer episodio de la serie ya somos conscientes de la importancia de todos estos rasgos en cada personaje. Así, tras el ingreso de Piper Chapman en prisión, somos testigos de una reveladora escena entre dos latinas y la protagonista en la que una de las primeras, que lleva más tiempo en prisión, reprocha a la otra, recién llegada, que no hable español, y se apoya para reforzar su argumento en el hecho de que incluso Chapman, una mujer blanca y también nueva allí, sí lo sepa utilizar:

Ejemplo 5. OITNB 01x01 (31:47/34:16)

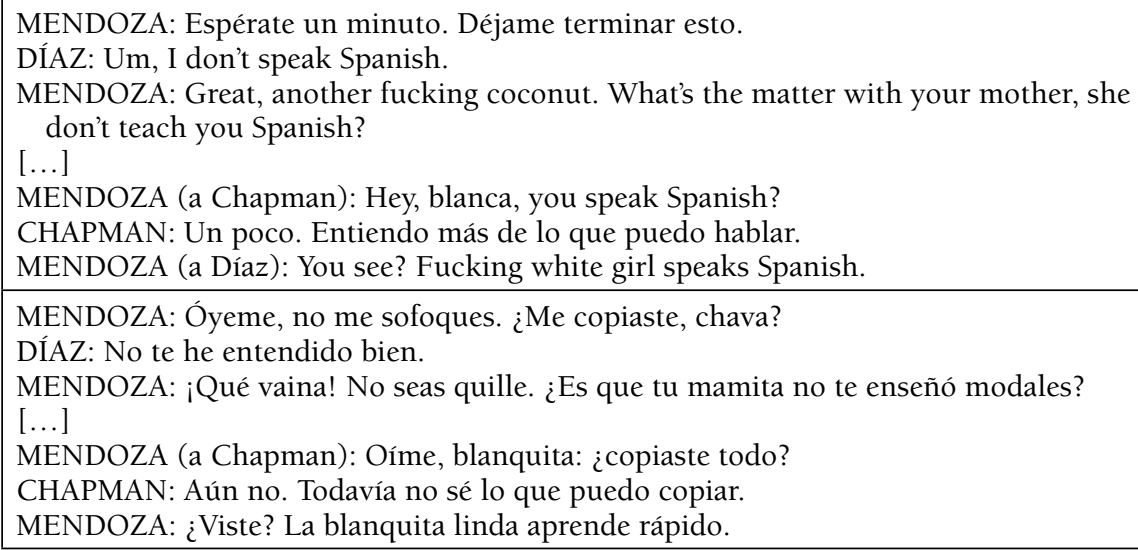

La conversación y su contenido son especialmente relevantes por distintas razones en nuestro trabajo. En primer lugar, porque en la versión original se asocia la identidad latina con el uso del español. Desde un enfoque sociolingüista, "language becomes a powerful tool in the display of the ethnic self, a tool that can either reinscribe or subvert the ethnic identities assigned by outsiders" (Bucholtz 1995: 357). De este modo, la activación del español sirve como marcador de latinidad en un sentido similar a como explica Butler su teoría de la performatividad del género a través de actos discursivos concretos. Tal y como explica Fina (2007: 372):

Participants in social activities 'do' identity work and align with or distance themselves from social categories of belonging depending on the local context of interaction and its insertion in the wider social world. [...] Identity claims and displays are embedded in social practices and respond to a complex interplay of local and global factors. 
Una de las prácticas más habituales en la serie es el code-switching del grupo de latinas, es decir, la alternancia del inglés y el español por parte de estas reclusas bilingües en un mismo discurso. Así, localizar su aparición en los diálogos de la serie nos puede ayudar a descubrir distintos aspectos de la identidad de sus usuarias y comprender mejor a los personajes.

En este sentido, las presas de la comunidad latina no son un grupo homogéneo, y la lengua compartida para sus interacciones habituales es el inglés. Sin embargo, cabe destacar que surgen determinados contextos en los que existe cierta tendencia a cambiar al español. Estos momentos suelen producirse en diálogos con familiares o amigos próximos sobre temas muy personales, como el siguiente caso, cuando Gloria, una portorriqueña que acaba en prisión, habla con su tía Lourdes sobre el maltrato que sufre por parte de su pareja:

Ejemplo 6. OITNB 02x05 (16:02)

LOURDES: Llama a la policía. Que deporten al cabrón ese.

GLORIA: Olvídate de la policía. Lo único que van a traer son más problemas.

LOURDES: ¿Y esto qué es? ¿La felicidad eterna? Piensa en tus hijos.

GLORIA: Él nunca les ha puesto la mano encima. Arturo siempre los ha tratado bien.

LOURDES: Llama a la policía. Que deporten al cabrón ese.

GLORIA: Olvídate de la policía. Un día te van a traer problemas.

LOURDES: ¿Y esto qué es? ¿La felicidad eterna? Piensa en tus hijos.

GLORIA: Él nunca les ha puesto la mano encima. Arturo siempre les ha tratado bien.

El original emplea el español, y esta alternancia destaca especialmente en la versión original y contribuye a dar forma a la identidad, pero en el paso al español el espectador meta no es consciente de este cambio de código. En este sentido, cabría plantearse junto a Herrera (en Martínez-Sierra et al. 2010: 16) la pertinencia de la estrategia de doblaje en productos audiovisuales políglotas sin recurrir a otras técnicas como las que propone el marco de trabajo de Martínez-Sierra et al. (2010).

Cada una de las lenguas se emplea, de este modo, para construir una parte fundamental de las identidades de las presas latinas, de manera que la alternancia entre las lenguas no es casual ni neutra, tal y como vemos en la combinación bilingüe en el siguiente diálogo entre la tía y los hijos de Gloria: 


\section{Ejemplo 7. OITNB 02x05 (17:27)}

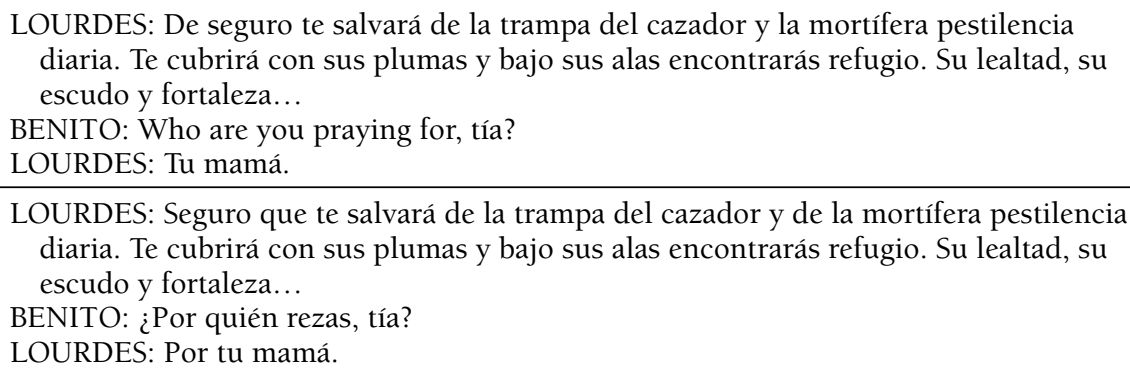
diaria. Te cubrirá con sus plumas y bajo sus alas encontrarás refugio. Su lealtad, su escudo y fortaleza...

LOURDES: Seguro que te salvará de la trampa del cazador y de la mortífera pestilencia diaria. Te cubrirá con sus plumas y bajo sus alas encontrarás refugio. Su lealtad, su escudo y fortaleza...

BENITO: ¿Por quién rezas, tía?

LOURDES: Por tu mamá.

En este caso, Lourdes, una santera que ofrece sus servicios en la trastienda del local de gloria, reza en español ante su sobrino nieto, que interacciona con ella en inglés. En la versión original, las creencias católicas y relacionadas con la magia y la santería de Lourdes solo surgen en español, mientras que en la versión doblada dicha relación se difumina al traducir todo el diálogo en castellano, sin diferenciar el cambio de lengua entre tía y sobrino. De esta forma, asumimos que la percepción de la identidad latina para el público español no será la misma que se pretende transmitir en la versión original y que, en cierto modo, la imagen presentada del personaje de Lourdes cambia en el doblaje.

En la versión original también es común el salto al español en los momentos de mayor tensión, cuando se desatan peleas o enfrentamientos. De este modo, como ejemplo, escuchamos un diálogo en el que surge el español en la escena en la que Marisol (mexicana) y María (dominicana) se pelean mientras preparan la comida de la cárcel. En este caso, el inglés se convierte en el punto de partida desde el que incluir términos en español o diálogos bilingües. La traducción de estos lenguajes híbridos podría funcionar como una herramienta para cuestionar el poder de las culturas y lenguas dominantes $y$ visibilizar otras comunidades que habitan en el in-between, en palabras de Bhabha (1994: 1-2), localizadas en una situación dual que mira hacia la cultura dominante sin olvidar su origen latino. Por ello, la opción doblada únicamente al español, de forma monoglósica, sin ningún componente híbrido, describe, desde nuestro punto de vista, un escenario diferente al que presenta la versión original. Valga como ejemplo la siguiente escena en la que Marisol y María, reclusas latinas, mantienen una conversación en inglés acerca de unos nuevos puestos de trabajo que se van a ofrecer para las presas, y en la que, cuando el tono aumenta, acaban por recurrir al español: 


\section{Ejemplo 8. OITNB 03x05 (06:16)}

MARISOL: They don't pay that much for dummy work. Nah, whatever it is, it’s gonna
take skills and smarts.
MARÍA: Yeah, what're you gonna wow them with? Your eyeliner skills, or your smarts
about how many words rhyme with "bleak" in emo songs?
MARISOL: Fuck you and your stupid sad face. We didn't take your baby away, okay?
MARÍA: Yeah? You should shut up about shit you don’t know about.
MARISOL: You should shut up, you know. Hey! At least I got ambition. Qué barbera.
MARÍA: ¿Ambición? Eso es lo que tú llamas a decir: ¡ay, mira allá, hay algo shiny!
MARISOL: ¿Quieres empezar tú? ¡Órale wey!
MARÍA: ¡Egoísta! ¡No me toques!
MARISOL: No pagan tanto por un trabajo tan tonto. Sea lo que sea, habrá que ser
capaz y lista.
MARÍA: ¿Y con qué los quieres impresionar tú? ¿Con tu capacidad con el delineador o
Con las palabras que riman con "desolador" en las canciones emo?
MARISOL: Que te den a ti y a tu cara de triste. Nosotras no te hemos quitado a tu hija.
MARÍA: ¿Ah no? Cierra la boca sobre ciertas cosas.
MARISOL: Ciérrala tú. Yo tengo ambición. Bésame el culo.
MARÍA: ¿Ambición? ¿Esa palabra la acabas de aprender? Eh mira allí, algo que brilla.
MARISOL: ¡No me eches a mí tu mierda!
MARÍA: ¡Zorra egoísta! ¿Qué? ¿Qué?

Somos conscientes de los problemas específicos que plantea la traducción audiovisual en estas situaciones; no obstante, la traducción completa en español sin ninguna marca que diferencie el salto que se aprecia en la versión original supone, desde nuestro punto de vista, la pérdida de matices que definen a los diferentes personajes. Además, se emplea, a lo largo de toda la serie y dentro de la clasificación que establece Martínez-Sierra et al., un español estándar que no se corresponde con el acento de ninguna de las reclusas y que supone una estrategia domesticadora (Martínez-Sierra et al. 2010: 21) que puede llegar a conseguir una esencialización de las identidades latinas en el sistema penitenciario estadounidense.

En otras ocasiones se observa además cómo las reclusas latinas recurren al español cuando se enfrentan a otras presas que pertenecen a otros grupos raciales o étnicos que no hablan ni entienden el español. En el siguiente ejemplo, el sistema de desagüe de los baños que utilizan las latinas se estropea y se ven obligadas a ducharse en los que utilizan las presas negras. Tras la conversación entre las dos líderes de ambos grupos, Gloria y Vee, se produce un conato de pelea, desencadenado precisamente por el cambio al español de una de ellas. El componente subversivo se omite en este caso en la versión doblada, pues se diluye en la versión completamente en español, sin que se llegue a reproducir el intercambio de rebelión y dominación implícito que sí se aprecia en el original. El poder que comunica el cambio de código durante la discusión 
se pierde absolutamente en la versión doblada con un diálogo neutro que en ningún caso plantea un intento de oposición al orden hegemónico:

Ejemplo 9. OITNB 02x05 (02:29)

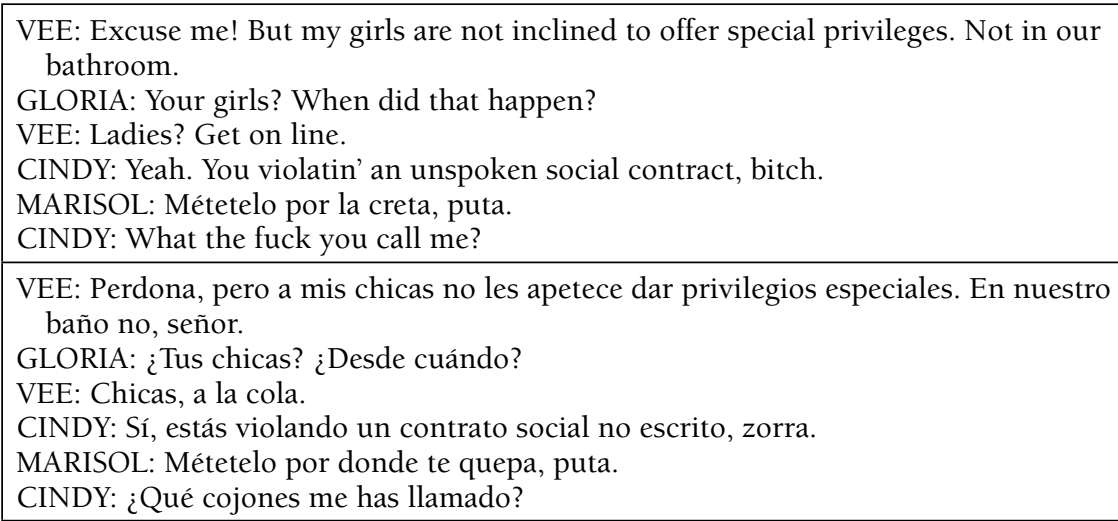

De este modo, observamos que en la versión original se aprecia un cambio de idioma en función del diálogo, de su contenido y de sus participantes que definirán la identidad de cada una de las latinas para el público. Sin embargo, esa alternancia entre el español y el inglés y el uso de términos en español propios de dialectos latinos no se traslada en ningún caso a la versión doblada para el mercado español, puesto que ambas lenguas quedan unificadas en la versión en castellano para España. Especialmente reparamos en que los asuntos más personales y delicados se tratan, en general, en el original, a través del español o incluyendo saltos con el inglés dentro del grupo de latinas. Este cambio de código contribuye a definir de manera determinante la identidad ya que, en función de lo que se quiera trasmitir y del tema sobre el que hablen, se recurre a una lengua o a otra. Además, resulta llamativo que, frente a la importancia del español y del grupo latino en la versión original, no se haya optado por soluciones de doblaje o subtitulación híbridas inglés/español como las que comprenden los marcos de trabajo de Martínez Sierra et al. (2010) y de HigesAndino et al. (2013), más afines al paradigma interseccional que propone la serie, tal y como sí se hace con otras lenguas mucho menos frecuentes en los capítulos. Así, en el siguiente ejemplo, observamos el papel de otras lenguas y su relevancia a la hora de construir la identidad (sexual) de las presas. En este caso, en la versión doblada al español se ha mantenido prácticamente íntegro el fragmento en alemán con subtítulos abiertos en español para una escena de alto contenido sexual entre dos mujeres: Poussey (estadounidense que vive en Alemania) y Franzi (alemana). 
Ejemplo 10. OITNB 02x06 (18:30)

\author{
POUSSEY: Dort? \\ FRANZI: Höher, vielleicht \\ POUSSEY: Warte, Bein Krampf! \\ FRANZI: Sorry. Okay... Das ist... Das ist gut... \\ POUSSEY: Ja? \\ FRANZI: Ja... Nein, es ist weg. \\ POUSSEY: Ich habe dir gesagt, Scheren geht es nicht. \\ POUSSEY: Und? \\ FRANZI: Höher. \\ POUSSEY: Warte, Bein Krampf! \\ FRANZI: Perdona. Vale... Das ... Das ist gut. \\ POUSSEY: Ja? \\ FRANZI: Ja... Nein, das ist weg. \\ POUSSEY: Ich habe dir gesagt, Scheren geht es nicht. \\ Subtítulos abiertos en español \\ POUSSEY: ¿Algo? \\ FRANZI: Más alto quizás. \\ POUSSEY: Espera. // Calambre en la pierna. \\ FRANZI: Es agradable. // No. // Se ha ido. \\ POUSSEY: Te dije que lo de la tijera no servía.
}

El espectador español que contempla esta escena es plenamente consciente de la entrada de una segunda lengua en el desarrollo de la acción. La solución de traducción que se le ofrece es el subtitulado que, como recordamos según el análisis propuesto por Martínez-Sierra et al. 2010, se orienta hacia el extremo extranjerizante del espectro del eje domesticación-extranjerización. No obstante, debemos destacar que tal apreciación también sería pertinente en los casos en los que se intercala la lengua española que utilizan los personajes latinos por tratarse de una variedad diferente a la peninsular y, lo que es más importante, por condicionar una representación radicalmente diferente de sus identidades.

En contraposición a esta escena en alemán para el público meta, las referencias en español al sexo y a las sexualidades minoritarias son prácticamente inexistentes. Como hemos visto, el inglés es la lengua predominante para casi todas las intervenciones del grupo de las latinas, pero es evidente el cambio hacia el español cuando tratan de imponerse en un enfrentamiento y, especialmente, en el tratamiento de asuntos referentes al ámbito familiar y personal. Por este motivo resulta extraño escuchar el siguiente diálogo en la versión original entre dos de las latinas en inglés, a pesar de ser un momento íntimo entre ellas. En la siguiente escena, Maritza y Marisol, que llevan tiempo en 
prisión, se lamentan al final del día de San Valentín de que están malgastando su juventud sin posibilidad de mantener relaciones con hombres:

Ejemplo 11. OITNB 02x06 (52:37)

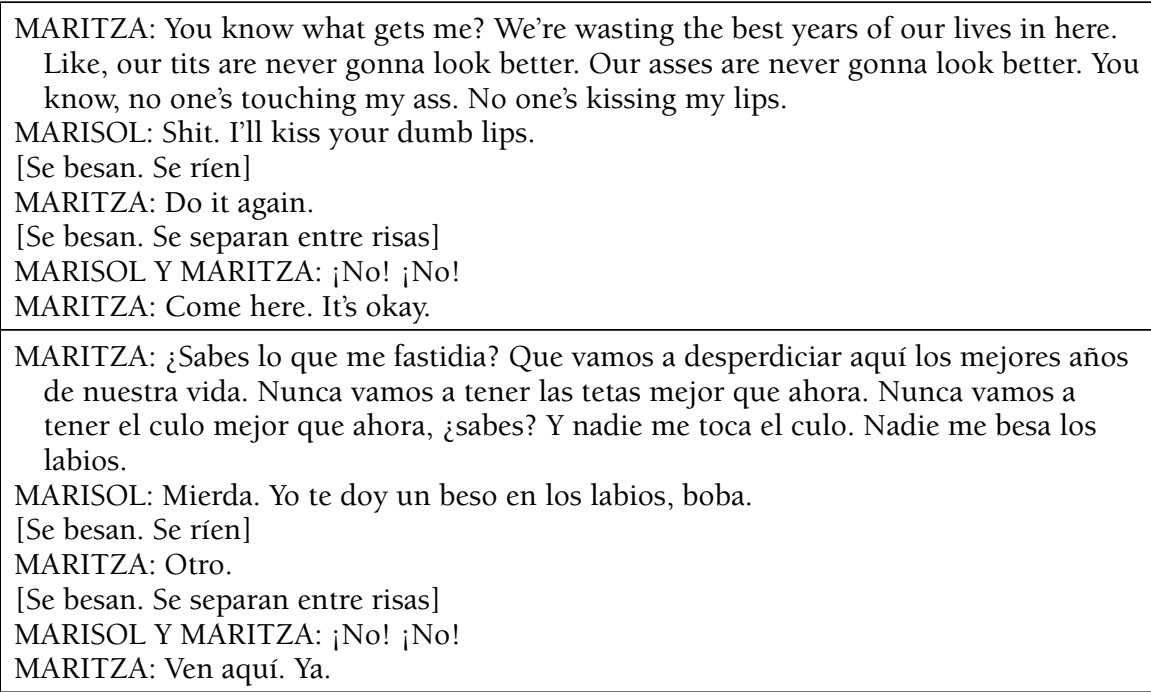

Este fragmento aporta información por distintos motivos. En primer lugar, los espectadores en versión original configuran la sexualidad de ambas a través del inglés, lo que aleja cualquier atisbo de disidencia sexual de su carácter latino, construido desde y a través del español. Por otra parte, esta conversación sobre prácticas sexuales minoritarias es la única que se produce entre latinas. En una serie que descompone el estereotipo y muestra personajes interseccionales, que el grupo de latinas no incluya ningún personaje bisexual $u$ homosexual puede llevar a una cierta esencialización de la identidad latina, más aún si pensamos que en el resto de grupos de presas negras, blancas, de mayor edad, de adictas a las drogas, así como en personajes más independientes entre las reclusas como Soso Brooks, estadounidense de ascendencia japonesa y escocesa, sí que hay ejemplos de minorías sexuales. Como recuerda acertadamente Mira (2012: 42), las representaciones narrativas de lesbianas construidas en los productos audiovisuales facilitarán la creación de paradigmas reconocibles y visibilizadores para este grupo, pero, si contienen prejuicios, "estos se perpetuarán entre los espectadores poco preparados o que no son conscientes de cómo se han generado los materiales de ficción". En esta misma línea, observamos que existe una identidad saliente, término que recuperamos desde el ámbito de la Psicología y que ya se ha aplicado en el estudio de la traducción 
de sexualidades minoritarias (Martínez Pleguezuelos 2016), que destaca en los personajes latinos y, en buena medida, reafirma los estereotipos que tradicionalmente han pesado sobre estos en televisión. En este caso, se aprecia que las líneas argumentales de las presas latinas giran en torno a temas familiares en mayor medida que en el resto de personajes, mientras que la sexualidad se convierte en casi un tema tabú entre ellas.

En el lado opuesto, entre las reclusas lesbianas y blancas, las relaciones sexuales con otras presas se tratan abiertamente y los ejemplos abundan en la serie. Tomemos por caso, como muestra, la competición entre Boo y Nicky, dos de las presas, para comprobar cuál de las dos conseguirá sumar más encuentros sexuales con mujeres dentro de la prisión:

\title{
Ejemplo 12. OITNB 02x04 (12:14)
}

\author{
BOO: What's this? \\ NICKY: Oh! Put that back, please. Would you give it back? \\ BOO: Who is Brook? Oh, yeah. That's the new girl, isn't it? Hot one of the Asian \\ persuasion? Oh, my God! I found your fuck book? \\ NICKY: Right. So what if it is? \\ BOO: Oh, you fuckin' junkie. \\ NICKY: All right. \\ BOO: No, I get this. Better pussy than smack. Right? \\ NICKY: It's not an addiction. It's a collection, all right? Some people collect buttons or \\ Taco Bell Chihuahuas, I collect orgasms. See, I'm all about giving. Look, I am like a \\ bean-flicking Mother Teresa. \\ BOO: You know what? This here is my kind of competition. It's on. Ooh! Bitch, this is \\ on! \\ BOO: ¿Qué es esto? \\ NICKY: Déjalo, por favor. ¿Me lo quieres dar? \\ BOO: ¿Quién es Brook? Ah, sí, es la nueva. ¿Esa asiática que está tan buena? ¡Oh, dios! \\ ¡He encontrado tu libreta de polvos! \\ NICKY: ¿Y si es eso qué? \\ BOO: Oh, iputa yonqui! \\ NICKY: Déjame. \\ BOO: No, si te entiendo. Mejor coños que caballo, ¿no? \\ NICKY: No es una adicción, es una colección. Hay quien colecciona botones o llaveros \\ de propaganda. Yo colecciono orgasmos. Solo doy felicidad. Soy como una puñetera \\ Madre Teresa de hacer dedos. \\ BOO: ¿Sabes qué? A mí me molan estas competiciones. Empezamos. ¡Uh, tía, ha \\ empezado!
}

Como comprobamos en la versión doblada, el contenido sexual se vuelca para el público en España sin restricciones ni censuras. La competición tratada en el ejemplo 13 abarca como posibles candidatas a todas las mujeres de la cárcel, guardias incluidas, y está mediada por Chang, una reclusa asiática miembro 
del grupo de las Golden Girls. Esto da muestra de la naturaleza interseccional del resto de grupos y de cómo la sexualidad conforma, junto a la raza o la edad, la identidad de todas las reclusas. Esto mismo ocurre en el ejemplo 14 a continuación. Chapman, la mujer joven y blanca que protagoniza la serie, quiere enterarse de los pormenores del concurso que se establece entre Nicky y Bo (también mujeres blancas), y que arbitra Chang:

Ejemplo 13. OITNB 02x05 (07:09)

Los personajes que intervienen en esta escena demuestran dos aspectos diferentes que tienen que ver directamente con la interseccionalidad. Por un lado, la participación como árbitro de una reclusa asiática y de la tercera edad dinamita la distribución racial y etaria que esencializa los discursos dominantes sobre ambos rasgos. Por otro lado, no podemos olvidar que Chang es una reclusa heterosexual, y su mediación en el concurso tumba, además, el eje de la sexualidad en tanto que resta peso al componente de la sexualidad que tan determinante era en producciones audiovisuales pasadas. 
En la comunidad de reclusas negras existen también mujeres lesbianas que conviven abiertamente con su orientación sexual a pesar de los prejuicios y el rechazo que encuentran dentro de su propio grupo. En la siguiente conversación, Vee, líder del Gueto, habla con Taystee, otra de las presas, acerca de la relación de esta con Poussey, una presa negra y abiertamente lesbiana con la que mantiene una relación platónica que no trasciende nunca hacia el plano físico.

Ejemplo 14. OITNB 02x04 (52:21)

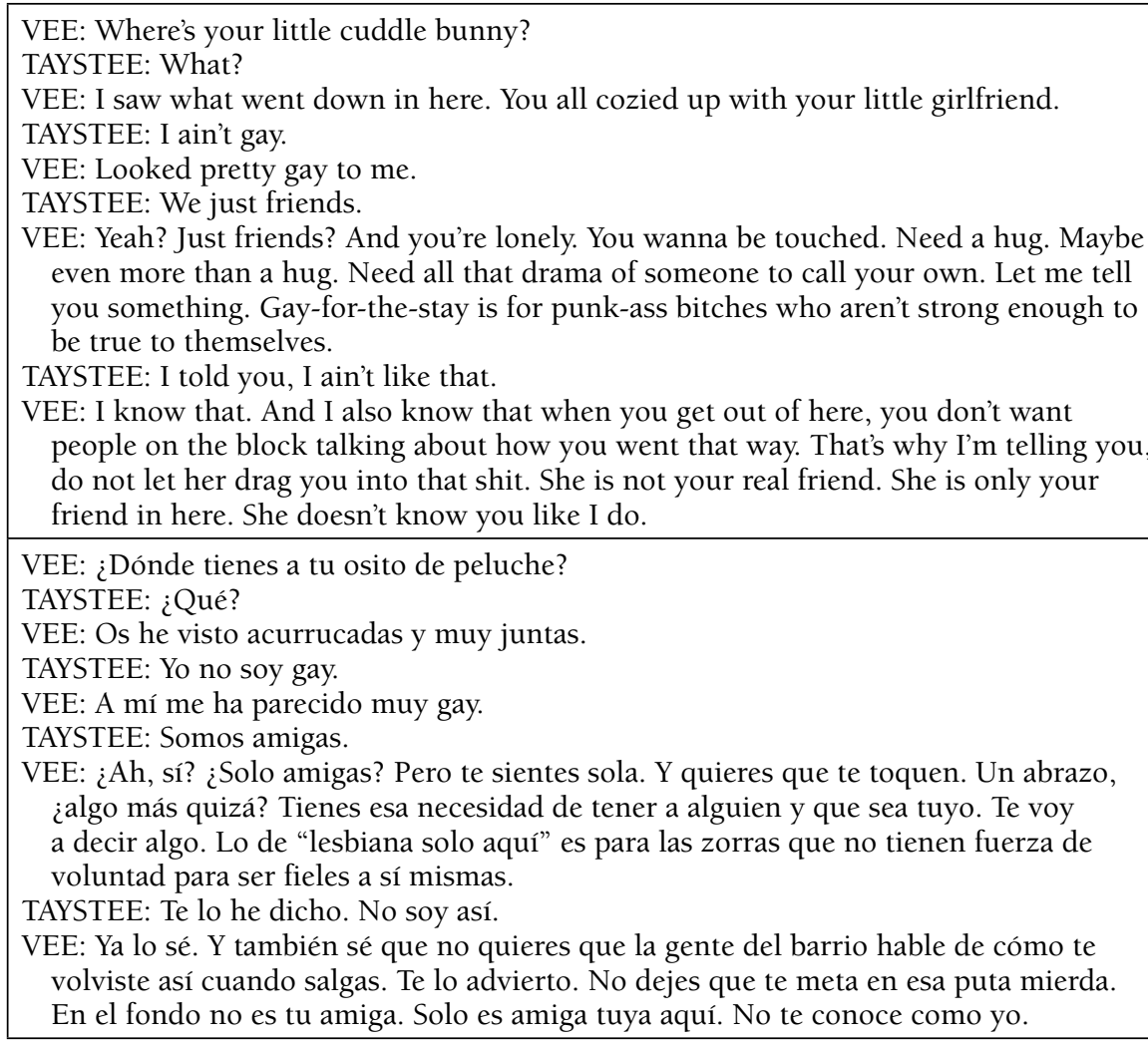

En todos estos casos, la traducción de OITNB funciona como la "escritura divergente" que propone Pérez L. de Heredia (2016a: 199) desde el "deviational translation" de Díaz-Cintas (2012b: 285). Tanto en la versión original como en la doblada al español, la serie conjuga en la mayoría de los grupos que conviven en la prisión de Litchfield distintas características que contribuyen a destruir los paradigmas identitarios esencialistas que, en gran medida, se proyectaban en la pequeña pantalla cuando el guion exigía la participación de mujeres 
negras, asiáticas, lesbianas o de mayor edad. De este modo, apreciamos capítulo a capítulo que la raza, la edad, la etnia, la lengua y la sexualidad son rasgos que moldean, de manera conjunta, la identidad de la mayoría de las presas.

\section{Conclusiones}

Como hemos comprobado en la investigación desarrollada en estas páginas, "absolute, unified and monolithic definitions of identity are no longer defensible and/or tenable" (House, Martín Ruano y Baumgarten 2005: 4), y así lo entendemos también en la construcción identitaria realizada en Orange Is the New Black. Distintas voces desde los Estudios de Traducción ya han defendido que las identidades interseccionales que se presentan en pantalla son el fruto de una compleja negociación discursiva que no entiende de estereotipos ni de nociones y etiquetas independientes como "mujer", "negra", "lesbiana" o "judía". No obstante, a la vista de los ejemplos presentados y a diferencia del resto de grupos de presas en OITNB, resulta más complicado encontrar el modelo interseccional que la serie propone entre las reclusas latinas.

La pérdida que supone la neutralización de la alternancia de código entre el inglés dominante y el español ofrece al espectador en España una imagen diferente, pues consideramos que el texto meta no refleja de forma tan nítida como en el original el vínculo entre la lengua española y la esfera privada y familiar de las presas que recurren al español para tratar aspectos más personales. En este sentido cabría preguntarse si la desaparición del code-switching por parte del grupo de reclusas latinas no se vería compensada de algún modo con el uso de una variante de acento latino para el mercado español, lo que, en cierta medida, podría contribuir a la formación de sus identidades mediante el refuerzo y visibilización del rasgo latino. Igualmente, haber optado por una de las estrategias de traducción menos domesticadoras planteadas por Martínez-Sierra et al. (2010) o De Higes-Andino et al. (2013) en el tratamiento de productos audiovisuales multilingües podría haber contribuido a la representación de una imagen más cercana al grupo de latinas del original para el público meta español.

Como vemos, no cabe duda de que los retos que plantea la traducción de productos audiovisuales multilingües dificultan en gran medida la labor del traductor. No obstante, en consonancia con los objetivos principales de la investigación desarrollada, cabría recordar que, además de las restricciones de carácter técnico que subyacen en la traducción audiovisual, resulta necesario tener en cuenta la representación identitaria que se entrega al público meta y cómo esta puede verse afectada por las diferentes opciones de traducción empleadas en el doblaje. 
Al mismo tiempo, y de manera paralela en la versión original y en la doblada, parece destacable la invisibilidad de la identidad sexual en las latinas de la serie y la heteronormatividad que rige, en general, la sexualidad de todas ellas en comparación con la variedad de tendencias sexuales y de género presente en el resto de grupos. Por ello, a la luz de la comparativa realizada entre la versión original y la doblada al español, consideramos que sería positivo recordar los riesgos que entraña una reescritura monolingüe y esencialista de discursos en los que la polifonía de voces y los (des)encuentros culturales y sociales contribuyen a configurar identidades televisadas que reflejan la pluralidad y el multiculturalismo de nuestras sociedades.

\section{Referencias bibliográficas}

BHABHA, Homi K. (1994) The Location of Culture. Londres \& Nueva York: Routledge. BRUfaU AlVIRA, Nuria. (2009) Traducción y género: propuestas para nuevas éticas de la traducción en la era del feminismo transnacional. Salamanca: Universidad de Salamanca. Tesis doctoral inédita.

Brufau Alvira, Nuria. (2010) "Interviewing Luise von Flotow. A New State of the Art." Quaderns. Revista de traducció 17, pp. 283-292.

BuCHOLTZ, Mary. (1995) "From Mulatta to Mestiza: Language and the Reshaping of Ethnic Identity." En: Hall, Kira \& Mary Bucholtz (eds.) (1995) Language and the Socially Constructed Self. Nueva York: Routledge, pp. 351-373.

BUTLER, Judith. (1990) Gender Trouble. Feminism and the Subversion of Identity. Londres \& Nueva York: Routledge.

CASTELLS, Manuel. (2008) "Comunicación, poder y contrapoder en la sociedad red (I). Los medios y la política." Telos 74. pp. 13-24Versión electrónica: $<$ https://telos.fundaciontelefonica.com/telos/articuloautorinvitado.asp@idarticulo= $=1 \&$ rev $=74$. htm>

Chambers, Samuel A. (2009) The Queer Politics of Television. Londres \& Nueva York: I. B. Tauris.

Cronin, Michael. (2006) Translation and Identity. Londres \& Nueva York: Routledge.

De Higes-Andino, Irene et al. (2013) "Subtitling Language Diversity in Spanish Immigration Films." Meta 58:1, pp. 134-145.

De MARCo, Marcella. (2012) Audiovisual Translation through a Gender Lens. Ámsterdam \& Nueva York: Rodopi.

DíAZ-CinTAS, Jorge. (2012a) "Presentation.” Meta 57:2, pp. 275-278.

DíAz-CinTAS, Jorge. (2012b) "Clearing the Smoke to See the Screen: Ideological Manipulation in Audiovisual Translation." Meta 57:2, pp. 279-293.

Díaz-CinTaS, Jorge; Ilaria Parini \& Irene RANZATo (eds.) (2016) Altre Modernità. Numero Speciale Ideological Manipulation in Audiovisual Translation. 
ENCK, Suzanne M. \& Megan E. Morrissey. (2015) "If Orange Is the New Black, I Must Be Color Blind: Comic Framings of Post-Racism in the Prison-Industrial Complex." Critical Studies in Media Communication 32:5, pp. 303-317.

FINA, Anna. (2007) "Code-switching and the Construction of Ethnic Identity in a Community of Practice." Language in Society 36:3, pp. 371-392.

Gentzler, Edwin. (2012) "Translation without Borders." En: Translation: A Transdisciplinary Journal. Versión electrónica: <http://translation.fusp.it/ articles/translation-without-borders>

GONZÁLEZ-IGLESIAS, J. David \& Fernando Toda. (2013) "Traducción y Conflicto: Perdidos en la traducción." En: Vidal Claramonte, África \& M. Rosario Martín Ruano (eds.) 2013. Traducción, política(s) y conflictos: legados y retos para la era del multiculturalismo. Granada: Comares, pp. 27-43.

HALl, Stuart. (1973) Encoding and Decoding in the Television Discourse. Birmingham: Centre for Cultural Studies, University of Birmingham.

Hall, Stuart. (1995) "The Whites of Their Eyes." En: Dines, Gail \& Jean M. Humez (eds.) 2003. Gender, Race, and Class in Media. A Text Reader. Londres \& Nueva Delhi: Sage, pp. 89-93.

HAll, Stuart. (1996) “Who Needs Identity?” En: Hall, Stuart \& Paul du Gay (eds.) 1996. Questions of Cultural Identity. Londres, Thousand Oaks \& Nueva Delhi: Sage Publications, pp. 1-17.

HaLl, Stuart. (2000) "The Question of Cultural Identity." En: Hall, Stuart; David Held; Don Hubert \& Kenneth Thompson (eds.) 2000. Modernity: An Introduction to Modern Societies. Malden \& Oxford: Blackwell, pp. 595-634.

House, Juliane; M. Rosario Martín Ruano \& Nicole Baumgarten. (2005) "Introduction." En: House, Juliane; M. Rosario Martín Ruano \& Nicole Baumgarten (eds.) 2005. Translation and the Construction of Identity. Seúl: IATIS, pp. 3-13.

MARTín RUANO, M. Rosario. (2016) "Traducción, medios de comunicación, opinión pública: los retos éticos de la construcción de la actualidad." En: Martín Ruano, M. Rosario \& África Vidal Claramonte (eds.) 2016. Traducción, medios de comunicación, opinión pública. Granada: Comares, pp. 1-14.

Martínez Pleguezuelos, Antonio Jesús. (2016) "Representación de la homosexualidad, identidad saliente y traducción: estudio del doblaje de Will \& Grace en español.” En: Martín Ruano, M. Rosario \& África Vidal Claramonte (eds.) 2016. Traducción, medios de comunicación y opinión pública. Granada: Comares, pp. 209-224.

MARTÍNEZ-SierRA, Juan José et al. (2010) "Linguistic Diversity in Spanish Immigration Films. A Translational Approach." En: Berger, Verena \& Miya Komori (eds.) 2010 Polyglot Cinema. Migration and Transcultural Narration in France, Italy, Portugal and Spain. Viena: LIT, pp. 15-28. 
MirA, Alberto. (2012) “¿Gay, queer, gender...? Paradigmas críticos. El ejemplo de representación lésbica en las nuevas series.” En: Vera Rojas, María Teresa (ed.) 2012. Nuevas subjetividades/sexualidades literarias. Barcelona \& Madrid: Egales, pp. 41-52.

Mulvey, Laura. (1975) "Visual Pleasure and Narrative Cinema." En: Brandy, Leo \& Marshall Cohen (eds.) 1999. Film Theory and Criticism: Introductory Readings. Nueva York: Oxford University Press, pp. 833-844.

PÉREZ L. DE HEREDIA, María. (2015) "Traducción y globalización de estereotipos interseccionales en televisión: una imagen panorámica.” En: Penas Ibáñez, María Azucena (ed.) 2015. La traducción. Nuevos planteamientos teóricos-metodológicos. Madrid: Síntesis, pp. 261-282.

PÉREZ L. DE HEREDIA, María. (2016a) "Reescritura divergente y traducción de estereotipos de género en televisión: el caso de Orange Is the New Black." En: Martín Ruano, M. Rosario \& África Vidal Claramonte (eds.) 2016. Traducción, medios de comunicación, opinión pública. Granada: Comares, pp. 193-208.

PÉREZ L. DE HerediA, María. (2016b) "Translating Gender Stereotypes: An Overview on Global Telefiction." En: Altre Modernità, número especial Ideological manipulation in Audiovisual Translation, pp. 166-181.

RosenberG, Alyssa. (2015) "How 'Orange Is the New Black' Wins at Illustrating Identity." En: The Washington Post. Versión electrónica: <https://www. washingtonpost.com/news/in-theory/wp/2015/09/22/how-orange-is-the-newblack-wins-at-illustrating-identity/?utm_term=.c24098bla31b>

SHOHAT, Ella \& Robert Stam. (2003) "Introduction." En: Shoat, Ella \& Robert Stam (eds.) 2003. Multiculturalism, Postcoloniality, and Transnational Media. New Brunswick, New Jersey \& Londres: Rutgers University Press, pp. 1-17.

VAlDiviA, Angharad N. (2014) "Latina/os and the Media: a National Category with Transnational Implications." Polifonia 21:29, pp. 13-27.

Vidal Claramonte, África. (2007) Traducir entre culturas: poderes, diferencias, identidades. Fráncfort del Meno: Peter Lang.

Vidal Claramonte, África. (2015) “Traducir al atravesado." Papers 100:3, pp. 345-363.

VON Flotow, Luise. (2009) "Contested Gender in Translation: Intersectionality and Metramorphics." Palimpsestes 22, pp. 245-256. Versión electrónica: <http://palimpsestes.revues.org/211> 


\section{NOTAS BIOGRÁFICAS / BIONOTES}

Antonio J. Martínez Pleguezuelos trabaja como profesor asociado en la Universidad de Alcalá. Es Licenciado en Traducción e Interpretación (U. de Granada), Máster en Traducción y Mediación Intercultural y Doctor en Traducción por la U. de Salamanca. Forma parte del Grupo de Investigación Reconocido "Traducción, Ideología y Cultura" de la U. de Salamanca, y sus líneas de investigación incluyen la traducción y la ideología, traducción e identidad y traducción y estudios de género y sexualidad, temas sobre los que ha publicado distintos capítulos y artículos.

JUAN DAVID GONZÁLEZ-IGLESIAS GONZÁLEZ es licenciado y doctor en Traducción e Interpretación por la Universidad de Salamanca. Su investigación gira en torno a los parámetros técnicos de los subtítulos y a la evolución diacrónica de los mismos. Sus principales líneas de trabajo son la traducción audiovisual, el subtitulado y la accesibilidad y la multiculturalidad en los textos audiovisuales Ha publicado artículos sobre los parámetros técnicos de los subtítulos y el multiculturalismo en la traducción audiovisual en diversas revistas internacionales. En la actualidad es profesor en el Grado de Traducción e Interpretación de la Universidad Autónoma de Madrid y traductor en ejercicio.

Antonio J. Martínez Pleguezuelos works as an Associate Lecturer in the University of Alcalá. He holds a BA in Translation and Interpreting (U. of Granada), a Master's Degree in Translation and Intercultural Mediation and a $\mathrm{PhD}$ in Translation (U. of Salamanca). He is part of the Official Research Group TRADIC "Traducción, Ideología y Cultura" of the University of Salamanca. His main research topics are Translation and Ideology, Translation and Identity, and Translation and Gender and Sexuality Studies. He has published several articles and book chapters in these fields.

JuAn DAVID GonZÁlez-Iglesias GonZÁlez holds a BA in Translation and Interpreting and $\mathrm{PhD}$ in Translation from the University of Salamanca. His research focuses on the technical parameters of subtitles and the evolution of those parameters along time. His main lines of research are audiovisual translation, subtitling and accessibility and multiculturalism in audiovisual texts. He has published articles on the technical parameters of subtitles and on multiculturalism in audiovisual translation in different international journals. Currently, he works as a lecturer in the Degree of Translation and Interpreting. 